\title{
Channeling Crystals for Positron Production
}

\author{
Franz-Josef Decker \\ Stanford Linear Accelerator Center; Stanford, California $9 \$ 309$
}

\section{Abstract}

Particles traversing at small angles along a single crystal axis experience a collective scattering force of many crystal atoms. The enormous fields can trap the particles along an axis or plane, called channeling. High energy electrons are attracted by the positive nuclei and therefore produce strongly enhanced so called coherent bremsstrahlung and pair production. These effects could be used in a positron production target: A single tungsten crystal is oriented to the incident electron beam within $1 \mathrm{mrad}$. At $28 \mathrm{GeV} / \mathrm{c}$ the effective radiation length is with $0.9 \mathrm{~mm}$ about one quarter of the amorphous material. So the target length can be shorter, which yields a higher conversion coefficient and a lower emittance of the positron beam. This makes single crystals very interesting for positron production targets.

\section{Introduction}

In linear $\mathrm{e}^{+}, \mathrm{e}^{-}$-colliders the positions have to be produced for every cycle, if no recirculation scheme is considered. At the positron source of the Staniord Linear Collider, SLC [1] a $30 \mathrm{GeV}$ electron beam of up to $5 \cdot 10^{10}$ particles strikes a tungsten target producing about 60 positrons. Four of these are captured with a pulsed high magnetic field [2], accelerated to $210 \mathrm{MeV}$ and brought back to the damping ring. After the damping ring the overall yield (= number of positrons/number of electrons on the target) is about 0.7 , below the desired value of 1.0 or more for tuning margins.

The main desired features of a positron source can be characterized by the following five points: It should produce many positrons (1) with a low emittance (2) and also polarization (3) would be preferable. The target should stand the average and peak power (4) with the high mechanical stresses, while a capture region should provide very bigh guiding magnetic fields (5).

Other ideas, like using a helical undulator for VLEPP [3] or back-scattered Compton photons from a laser [4] address mainly the issue of polarization. Here the possibility of channeling crystals (see Fig. 1) as a positron source is investigated, (compare also [5], where a crystal is considered only for producing photons for a normal target).

Compared with a normal amorphous target, a crystal behaves differently: More positrons (1, compare numbers with the five desired points from above) are produced in a shorter radiation length (2, less gcattering provides smaller ernittance) and polarization (3, probably linear [6]) has been observed. The energy transfer of a particle to the crystal is less since it reacts with a whole string of around 1000 atoms. So the target will receive less power (4), while

-Work supported by the Department of Energy contract DEACO3-76SFoos 15 .

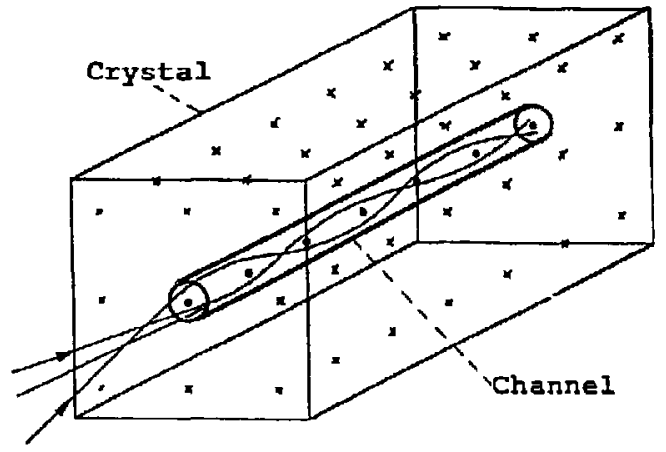

Beam particles

Figure 1: Principle of Channeling Crystal.

If a particle beam hits a crystal undar a small angle to one of its axes, a particie reacts with many atoms collectively and can therefore be trapped in a sma.l region around the axis (or plane), called channel. The large number of participating atoms (around 1000) generate enormous macro. scopic fields of the order of $E=10 \mathrm{TV} / \mathrm{m}$ (Tera-Volt) or $B=30 \mathrm{kT}$ (kilo-Tesla).

on the other hand a crystal (but out of tungsten) might be more sensitive. The enormous effective fields which guide the particles in channels are more than three orders of magnitude higher than the normally used fields (5) in accelerator physies. The main characteristics are summarized in the next section and afterwards the detailed target considerations are discussed.

\section{Channeling Characteristics}

The phenomenon of the channeling of particles in crystals is a wide field of physics; an overview and more introductions are given in [7] and references therein. The different behaviors with the energy of the particles, the characteristic angles of the beam to the crystal, the different kinds of particles $\left(\mathrm{e}^{+}, \mathrm{e}^{-}, \gamma\right)$ and corresponding theoretical approaches are described.

\subsection{Different Particle Energies}

At low energies only a few transverse energy levels are quantum mechanically allowed in the potential valley of height $U_{0}$ between crystal axes. At higher energies the

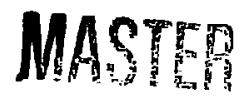

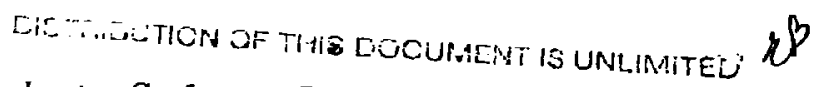

Presented at the IEEE Particle Accelerator Conference, San Francisco, CA, May 6-9, 1991 
particles get heavier and more levels are possjble, till it goes to a quasi-classical continuum.

\begin{tabular}{|l|c|c|}
\hline & Particle Energy & $\gamma$-Emission \\
\hline $\mathrm{a}:$ & low $\approx 10 \mathrm{keV}$ & Characteristic line spectrum \\
$\mathrm{b}:$ & med. $\approx 10 \mathrm{MeV}$ & $n \quad$ (more lines) \\
$\mathrm{c}:$ & high $\approx 10 \mathrm{GeV}$ & Classical synchrotron radiation \\
\hline
\end{tabular}

\subsection{Important Angles at High Energies}

Different characteristic angles influence the behavior of the beam at $25 \mathrm{GeV}$ (compare Fig. 2):

1. Characteristic angle: $\theta_{0}=\frac{U_{0}}{m_{0} c^{2}} \approx 0.5-1.0 \mathrm{mrad}$.

2. Lindhard ıngle (maximum angle being kept in channel): $\psi_{L}=\sqrt{\frac{2 U_{0}}{\gamma m_{0} c^{2}}} \approx 100-200 \mu \mathrm{rad}$.

3. Gamma or pair production angle: $1 / \gamma \approx 20 \mu \mathrm{rad}$.

4. Beam divergence: $\sigma^{\prime}=\sqrt{\varepsilon / \beta} \approx 5 \mu \mathrm{rad}$, (at $\gamma \varepsilon=$ $\left.5 \cdot 10^{-5} \mathrm{mrad}, \beta=30 \mathrm{~m}\right)$.

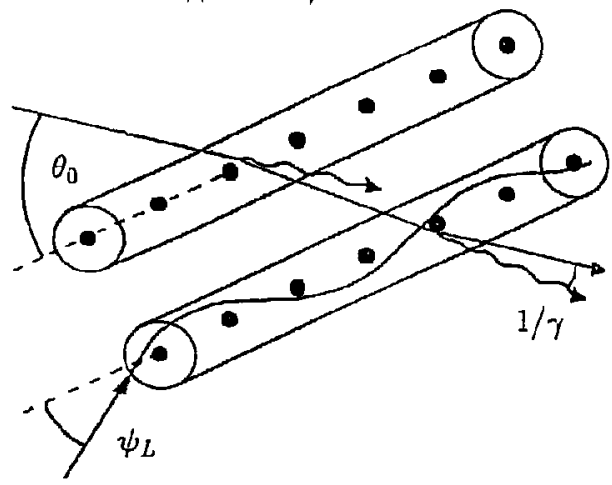

Figure 2: Angles in a Channeling Crystal.

Depending on energy different angles characterize the beam behavior in a crystal.

\section{$2.3 \mathrm{e}^{+}, \mathrm{e}^{-}$or $\gamma$-Beams with $\psi \leq \psi_{L}$}

In amorphous material the three kinds of particles in a particle shower behave similialy. In aligned crystals however there are big differences: Electrons are channeled near the atoms causing lots of radiation, positrons are between atoms and photons are not channeled, but create lots of pairs below an angle of $\theta_{0}$.

\subsection{Theoretical Approaches}

Depending on the energies, angles and kinds of particles different theories have been developed $[8,9]$. The behavjor of a beam hitting a crystal at large angles to an axis or plane corresponds to an amorphous target and the normal Bremsstrahlung (BS) or for photons a pair production (PP) with a normal Bethe-Heitler rate (BH) occurs.
At smaller angles (around $\theta_{0}$ ) the interference between the crystal axes or planes enhances the $\gamma$-production by coherent Bremsstrahlung (CBS) or CPP for photons. At even smaller angles below $\psi_{L}$ (see section 2.2 ) channeling radiation (CR) occurs. It is different for electrons and positrons since electrons are trapped near the atoms while positrons are in the space between atoms. Although photons are not channeled, the corresponding channeling pair production (ChPP) [10] produce channeled pairs since $1 / \gamma<\dot{w}_{L}$.

For different crystal material the pair production enhancement over $\mathrm{BH}$ is twice at $120 \mathrm{GeV}$ for $\mathrm{Si}, 50 \mathrm{GeV}$ for $\mathrm{Ge}$ and $15 \mathrm{GeV}$ for $W[9]$, so it is only important at very high energies. Since the particles of high energies traverses along a large number of atoms, the fields can be averaged, leading to a constant field approximation (CFA). Photons create a pair in the field of the string rather than a single atom. The table below gives a rough comparison of the different theories with electrons in accelerator fields. A more detailed scenario of an electromagnetic shower is given in the next section.

\begin{tabular}{|c|c|c|}
\hline Pairs & Photons & Accelerator \\
\hline PP (BH) & BS & Bremsstrahlung \\
CPP & CBS & wiggler \\
CliPP & CR & undulator \\
C.FA & CFA & synchrotron light \\
\hline
\end{tabular}

\section{Target Consideration}

A positron production target should produce many position in a small angle. The different channeling consequences are briefly summarized. As material a tungsten crystal in $\langle 111\rangle$ axis orientation is considered. At high energies an enhancement for pair production in $W$ is at $15 \mathrm{GeV}$ twice $\mathrm{BH}$ and at $30 \mathrm{GeV}$ about 3.2 times $\mathrm{BH}$. The shorter radiation length (normal is $3.5 \mathrm{~mm}$ ) of $0.9 \mathrm{~mm}$ at $28 \mathrm{GeV}$ leads to a shorter target length with less scattering (lower emittance) and less losses and therefore a higher yield $=N\left(\mathrm{e}^{+}\right) / N\left(\mathrm{e}^{-}\right)$. Channeled positrons scatter less than electrons resulting in more yield and a smaller angle. Polarization has been observed.

Single tungsten crystals can be made up to $10 \mathrm{~cm}$ long by $5 \mathrm{~mm}$ diameter with a small mosaic spread of about $120 \mu \mathrm{rad}$ [11]. It is assumed that a similar temperature and mechanical behavior of the amorphous tungsten metal will be found with single tungsten crystals.

\subsection{Detailed Shower Evolution}

The following scenario of the $\mathrm{e}^{t}, \mathrm{e}^{-}, \gamma$-shower of a $30 \mathrm{GeV}$ $\mathrm{e}^{-}$-beam in an oriented $\langle 111\rangle$ tungsten crystal is expected: At the higher $\mathrm{e}^{-}$-energies $(30 \mathrm{GeV})$ about three times as much photons have been measured after $1 \mathrm{~mm}$ length [14]. At small angles $\left(<\psi_{L}\right)$ the higher energy photons ( $>15 \mathrm{GeV}$ ) may contribute to an enhanced pair production by ChPP (hard shower [9]). At lower energies there is still CBS and more photons $N_{\gamma}$ than charged particles $N_{e}$ are present, $N_{\gamma} / N_{e} \approx 11$ [8]. These photons lead via $\mathrm{BH}$ to a normal pair production (only $\gamma$-production is enhanced $=$ soft shower). 
At lower energies the opening angle becomes as big as the Lindhardt angle and $\theta_{0}$ (compare section 2.2):

$$
2 / \gamma=\psi_{L}=\sqrt{\frac{2 U_{0}}{\gamma m_{0} c^{2}}}=\frac{U_{0}}{m_{0} c^{2}}=\theta_{0}=0.8 \mathrm{mrad}(\text { for } \mathrm{W}) .
$$

This energy of about $1.3 \mathrm{GeV}$ occurs after about three effective radiation lengths. At this point in the shower the enhancement of the crystal decreases and a second, normal target could be considered from then on [5].

But also another effect of the crystal can be used: Low energy channeled positrons scatter about ten times less with the crystal [15] and will leave it with a small emittance (see bulow). Additionally there are transmissions from free states in transverse motion to bound states [15] insreasing the number of channeled positrons. They ascillate like in a helical undulator and lower their transverse energy states via characteristic channeling radiation. This damps their effective transverse emittance. It is interesting to look at the normalized emittance $\gamma \varepsilon$ of the channeled particles. Wilh $\varepsilon=\sigma^{2} / \beta=\beta \sigma^{\prime 2}$ and assuming a low betatron function of $\beta=0.1 \mathrm{~m}$ and $\sigma^{\prime}=\psi_{L}^{\prime}$, it follows

$$
\gamma \varepsilon=\gamma \beta \frac{2 U_{0}}{\gamma m_{0} c^{2}}=2 \beta \theta_{0}=16 \cdot 10^{-5} \mathrm{mrad} .
$$

The angular part is independent of energy, while the spatial part

$$
\gamma \varepsilon=\gamma \frac{\sigma^{2}}{\beta}=16 \cdot 10^{-5} \mathrm{mrad} \quad(\text { for } \gamma=16, \sigma=1 \mathrm{~mm} \text { ) }
$$

decreases with energy, if the particles could be kept in the same channel. At very low energies, where $1 / \gamma>\psi_{L}$, only a part of the positrons will be channeled immediately.

\subsection{Beam Power and Polarization}

Beam Power on Crystal The critical topic is the damage of the crystal by heat or stress and any lattice defects by radiation, which needs futher experimental investigations. Fluxes of up to $10^{15}$ particles causes no noticeable radiation damage [12]. Since one particle reacts with a string of many atoms less power is transferred to the target atoms. The vibration of single atoms round the string causes drrhanneling of the beam particles. If the target is cooled e.g. by liquid nitrogen even less energy is transferred and more pairs are produced [16]. The observed $\mathrm{Cu}$ crystals near the SLC target may lead to the hypothesis that an oriented crystal might be the most stable configuration of atoms in an environment of directed radiation.

Polarization Different hints and discussion of polarization have been reported $[6,16,17]$. A planar channeled particle will radiate linear polarized photons. The desired circular polarization would be achieved, if the particles could be trapped around an axis in an oriented helical mo tion. Helix-like crystals or crystals deformation of about $1 \mu \mathrm{m}$ wavelength might support such a motion. Or a normal crystal is oriented in such a way that the beam direction, the crystal axis and one crystal plane build a leftright asymmetry leading to different amounts of polarized photons.

\section{Outlook and Conclusion}

The enormous potentials in and of single crystals have been already and will be used more frequently in eccelerator physics. With bending crystals SSC-energies could be kept in a PEP-like tunnel [18] and a TeV linear collider could be built within $10 \mathrm{~m}$ [13]. By starting small projects lilie a positron target, the confidence and experience in this quiti new technology will grow. The feasibility and advantages of single crystals as a positron source have been discussed.

\section{References}

[1] Stan Edklund, The Stanjord Linear Collider Positron Target, SLAC-Pub-4437 Oct. 1987 or Workshop of Intense Positran Beams, Idaho, June 1987.

[2] A.V.Kulikov, S.D. Ecklund, E.M. Reuter, SLC Positron Flux Coneentrator, PAC San Francisco, May 1991.

[3] T.A. Vsevolozhskaya ot al., Helieal Undulator for Conversion System of the VLEPP Project, Preprint B6-129-INP, Novossibirsk.

[4] J.E. Spencer, High Brightness Sources for Colliders, PAC, San Franciseo, May 1991.

[5] R. Chebab, F. Couchot, A.R. Nyajeh, F. Richard, X. Artru Study of a Positron Souree Generated by Photons from Ultrareiativistic Channeied Parlicles, LAL-RT 89-01 or PAC, Chicago, March 1989.

[6] R. Medenwaldt, S.P.Mpller, A.H. Sprensen et al, Investigetions of the coherent hard photon yields from $(50-900) \mathrm{GeV} / \mathrm{c}$ electrons/positrons in the strong crystalline fields of diamond, silicon end germanitm crystals (and W). CERN/SPSC 90-31, SPSC/P234 Add. 3, Oct. 1990.

[7] A.H.Sørensen and E.Uggertisj, The Channeling of Electrong and Positrons, Scientific American, 96pp., June 1989.

[8] V.N.Baier, V.M.Katkov and V.M.Strakhovenko, Cascade Processes in the Fields of the Axes of Aligned Sirlle Crystals, Nucl. Instrum. Methods B27, 360pp., 198r.

[9] R.Medenwaldt, S.P.Moller, S. Tang-Petersen et al., Detailed Investigations of Shower Formation in Ge- and W-Crystals traversed by $40-287$ GeV $/=$ Electrons, Physics Letters B, Vol. 227, number 3.4 Aug. 1989.

[10] A.H. Sørensen, E. Uggerhor, J. Bak and S.P. Møller, New A nal gais of the Two Competing Processes: Chanzehing- and Coherent Pair Production and a Comparison with Experimental Data, CERN-EP/84-149, Nov. 1984.

[11] Edited by R.A. Carrigan, Jr. and J.A.Ellison, Relativistic Chenneling. Workshop Maratea, NATO ASI Series: B, Physics, Vol. 165, Plenum Press, 1987, A.Seeger, Growing Large IIighly Perfect Single Crystals and its Limitations, $423 \mathrm{pp}$.

[12] Same book, S.I. Baker, Radiation Demage effects in Channeling Applieations, 391pp.

[13) Same book, P. Chen, Channeled Particle Acceleration by Plasme Waves in Metals, 517pp.

[14] V.N. Bajer, V.A. Baskov, V.B.Tanenko, Radiation by s8-GeV Electrons in a Thiek Tungsten Crgatal, JETP Lett., Vol. 49, No. 10, May 1989.

[15] A.H. Sarensen and E. Uggerher, Chenneling and Channeling Radiation, Nature, Vol. 32522 Jan. 1987.

[16] V.N. Baier, V.M.Kalkov and V.M.Strakhovenko, Theory of Pair Creation in Aligned Single Crystols, Nucl. Instrum. Methods B16, 5-21, 1986.

[17] U. Timm, Coherent Bremsstrahlung of Electrons in Crystals, Fortschritce der Physik 17, 765-808, 1969.

[18] Bending Beams by Crystals, CERN Courier, 5pp. May 15 o. 